\title{
ANÁlISE COMBINATÓRIA: METODOLOGIA DE APOIO AO PROFESSOR
}

\author{
COMBINATORIAL ANALYSIS: TEACHER SUPPORT METHODOLOGY
}

ANÁLISIS COMBINATORIO: METODOLOGÍA DE APOYO AL PROFESOR

Antonio Luís Venezuela ${ }^{1}$ (D) 9

\begin{abstract}
RESUMO
Probabilidade e a Análise Combinatória foram desenvolvidas em meados do século XVII. Pesquisas atuais realizadas por diversos autores concluíram que existem deficiências no ensino de Análise Combinatória devido às dificuldades que os professores apresentam em relação ao uso e ensino dessa teoria. Em muitos casos, o ensino se limita quase sempre ao treinamento no uso de fórmulas e algoritmos para encontrar o número de arranjos, combinações ou permutações, sem proporcionar a possibilidade dos alunos derivarem as referidas fórmulas pelo uso da manipulação dos elementos dos respectivos espaços amostrais dos problemas. Neste trabalho, a partir do plano de aula, a atuação do professor foi dividida em dois momentos: antes da aula e durante da aula. Propõe-se aqui uma metodologia que dá apoio ao professor no momento anterior à aula, quando ele está preparando o conteúdo a ser desenvolvido em classe. Para cada tipo de problema de combinatória foram apresentadas as fórmulas e uma lista de suas características. A partir dessas características construiu-se um fluxograma a fim de facilitar a resolução de diversos problemas de combinatória, utilizando, para isso, perguntas e respostas. Por fim, foram resolvidos, por meio desse fluxograma, diversos problemas extraídos da literatura.
\end{abstract}

Palavras-chave: Planejamento de aula. Ensino de Combinatória. Fluxograma.

\begin{abstract}
Probability and Combinatorial Analysis were developed in the middle of the 17th century. Current research carried out by several authors concluded that there are deficiencies in the teaching of Combinatorial Analysis due to the difficulties that teachers have in relation to the use and teaching of this theory. In many cases, teaching is almost always limited to training in the use of formulas and algorithms to find the number of arrangements, combinations or permutations, without providing the possibility for students to derive these formulas by using the manipulation of the elements of the respective sample spaces. In this work, from the lesson plan, the teacher's performance was divided into two moments: before class and during class. A methodology is proposed here that gives support to the teacher before the class, when he is preparing the content to be developed in class. For each type of combinatorial problem, formulas and a list of their characteristics were presented. Based on these characteristics, a flowchart was constructed in order to facilitate the resolution of various combinatorial problems, using questions and answers for this. Finally, several problems extracted from the literature were solved through this flowchart.
\end{abstract}

Keywords: Lesson planning. Combinatory Teaching. Flowchart.

\section{RESUMEN}

La probabilidad y el análisis combinatorio se desarrollaron a mediados del siglo XVII. Investigaciones actuales realizadas por varios autores concluyeron que existen deficiencias en la enseñanza del Análisis

\footnotetext{
1 Doutor pela Escola de Engenharia de São Carlos-Universidade de São Paulo (EESC-USP). Docente do Departamento de Física, Química e Matemática (DFQM) da Universidade Federal de São Carlos (UFSCar), Sorocaba, São Paulo, Brasil. Endereço para correspondência: Rod. João Leme dos Santos, km 110 - SP-264, Bairro do Itinga - Sorocaba, São Paulo, Brasil, CEP 18052-780. E-mail: alvenez@ufscar.br
} 
Combinatorio debido a las dificultades que tienen los docentes en relación al uso y enseñanza de esta teoría. En muchos casos, la docencia casi siempre se limita a la formación en el uso de fórmulas y algoritmos para encontrar el número de arreglos, combinaciones o permutaciones, sin brindar la posibilidad a los estudiantes de derivar estas fórmulas utilizando la manipulación de los elementos de los respectivos espacios muestrales. En este trabajo, a partir del plan de lección, la actuación del profesor se dividió en dos momentos: antes de clase y durante la clase. Se propone aquí una metodología que da soporte al docente antes de la clase, cuando está preparando los contenidos a desarrollar en clase. Para cada tipo de problema combinatorio se presentaron fórmulas y un listado de sus características. A partir de estas características, se construyó un diagrama de flujo con el fin de facilitar la resolución de diversos problemas combinatorios, utilizando preguntas y respuestas para ello. Finalmente, a través de este diagrama de flujo se resolvieron varios problemas extraídos de la literatura.

Palabras clave: Planificación de las clases. Enseñanza combinatoria. Diagrama de flujo.

\section{INTRODUÇÃO}

A Análise Combinatória é o ramo da Matemática que estuda e desenvolve procedimentos para resolver problemas de contagem de elementos de um conjunto com propriedades específicas. Além disso, ela estuda a formação e as propriedades dos agrupamentos que podem ser formadas, segundo determinados critérios, com os objetos de uma coleção. Existem, fundamentalmente, três espécies de agrupamentos: arranjos, permutações e combinações, e eles podem ser formados a partir de objetos distintos ou repetidos.

Desde os primórdios da humanidade existiram problemas de contagem, como, por exemplo, o problema 79 do Papiro de Ahmes (aproximadamente 1650 a.C.), a combinação de símbolos usados para adivinhações, encontrado no livro das Permutações (ou I-King) de WönWang (1182-1135 a.C.) (EVES, 2011) e o método que se assemelha ao Triângulo de Pascal, exposto no livro Brihat-Samhita de Varāhamihira, séc. VI (BOYER, 2012).

Blaise Pascal (1623-1662) impulsionou o desenvolvimento da Probabilidade com seu livro “Traité du Triangle Arithmétique”, em 1654 e, juntamente com Pierre de Fermat (16011665), com quem trocou diversas correspondências, são lançadas as bases da teoria da probabilidade. Inspirado pelos seus esforços, Christiaan Huygens (1629-1695) foi o primeiro a fazer um tratamento da Probabilidade como ciência, propriamente com a publicação de "De ratiociniis in ludo aleae", em 1657 (BOYER, 2012). Posteriormente, Jakob Bernoulli (16551705), em a “Ars Conjectandi”, publicado em 1713, fez uma exposição mais adequada a um novo ramo da matemática, que teve a contribuição de grandes nomes da matemática, como Abraham de Moivre (1667-1754), Daniel Bernoulli (1700-1782), Leonhard Euler (1707-1783) e outros (EVES, 2011).

Segundo Silva (2020), no Brasil, antes da década de 80, a Análise Combinatória já era 
parte do currículo da Educação Básica, porém somente para o Ensino Médio e para últimos anos do Ensino Fundamental. A partir da Lei de Diretrizes e Bases da Educação (BRASIL, 1996), um importante marco na Educação do Brasil, foram criados os PCNs para o Ensino Fundamental, em 1997, e, em 2000, para o Ensino Médio. Foi o início de uma reestruturação da educação do Brasil, que norteou a formação dos currículos em educação no país, em que houve uma ampliação do ensino de Análise Combinatória, passando a fazer parte $\operatorname{dos} 3^{\circ}$ e $4^{\circ}$ Ciclos do Ensino Fundamental.

Segundo a BNCC, Base Nacional Curricular Comum (BRASIL, 2018), no bloco temático Probabilidade e Estatística para o Ensino Médio, são sugeridas algumas habilidades que estão relacionadas à Análise Combinatória, a saber:

- EM13MAT310: Resolver e elaborar problemas de contagem envolvendo diferentes tipos de agrupamento de elementos, por meio dos princípios multiplicativo e aditivo, recorrendo a estratégias diversas como o diagrama de árvore,

- EM13MAT311: Resolver e elaborar problemas que envolvem o cálculo da probabilidade de eventos aleatórios, identificando e descrevendo o espaço amostral e realizando contagem das possibilidades,

- EM13MAT312: Resolver e elaborar problemas que envolvem o cálculo de probabilidade de eventos em experimentos aleatórios sucessivos,

- EM13MAT511: Reconhecer a existência de diferentes tipos de espaços amostrais, discretos ou não, de eventos equiprováveis ou não, e investigar as implicações no cálculo de probabilidades.

Moreira e Magina (2013) realizaram observações não sistemáticas de aulas sobre Análise Combinatória e verificaram que o ensino se limita, quase sempre, ao treinamento no uso de fórmulas e algoritmos a fím de encontrar o número de arranjos, combinações ou permutações, sem proporcionar que os alunos construam as referidas fórmulas por meio da manipulação dos agrupamentos formados por elementos de uma coleção de objetos. Ou seja, o professor apresenta as fórmulas e os alunos decoram, sem saber ao certo, em quais problemas irão usar cada uma. Além disso, a preocupação com qual fórmula a ser usada limita o aluno, no sentido de não possibilitar que procure compreender os textos dos problemas.

Como observou Sabo (2007), muitos professores de matemática, por diversas razões, evitam, ou até não abordam, de forma consistente, o tema Análise Combinatória. Alegam ser difícil ensinar, pois os alunos não têm capacidade de aprender algo tão sofisticado. Alguns afirmam que o tempo (ano letivo) é insuficiente e, então, torna-se necessário optar por outros 
temas que julgam mais importantes. Verificou também que alguns professores afirmam que eles próprios não têm esses conceitos construídos de forma sólida e significativa, e, por esse motivo, evitam abordar o tema ou, optam, apenas, por apresentar aos alunos um processo de aplicação de fórmulas prontas, sem justificativas ou explicações.

Schmidt e Mariani (2017) investigaram como são mobilizados os registros de representações semióticas em quatro dissertações que abordaram o desenvolvimento do raciocínio combinatório por meio de atividades didáticas, junto a alunos do ensino médio. Concluiu-se que as resoluções de problemas por meio de fórmulas de permutação, arranjo e combinação não configuraram uma escolha óbvia pelos participantes da pesquisa e, quando utilizadas, estiveram acompanhadas da regra do quociente e/ou regra do produto.

Nas aulas que envolvem Análise Combinatória no ensino médio, muitos professores encontram dificuldades ao desenvolver esse conteúdo, bem como em relação à resolução dos problemas dessa teoria. Mesmo expondo-se o Princípio Multiplicativo, mostrar ao aluno como se resolve todos os tipos de exercícios/problemas de combinatória é uma tarefa complicada. De acordo com Carvalho (2015), essa dificuldade aumenta quando, ao resolver os problemas de contagem não se faz uso do raciocínio simples. Considerando esse contexto, o planejamento das atividades é o melhor antídoto para a ansiedade e dificuldades que muitos professores sentem ao ensinar Análise Combinatória.

Planejar é analisar uma realidade específica, refletindo sobre as condições existentes, e prever as formas alternativas de ação para superar as dificuldades, ou alcançar os objetivos desejados. Portanto, o planejamento é um processo mental que envolve análise, reflexão e previsão. Já o plano é o resultado do processo mental de planejamento. O plano, confeccionado pelo professor é apenas um roteiro, um instrumento de referência e é esquemático. Ele deve ser personificado pelo professor, que o enriquece e lhe confere sua dinâmica (HAYDT, 2011).

Ao planejar e propor um plano de aula, entre outras coisas, o professor especifica os itens e subitens do conteúdo que serão trabalhados durante a aula e define os procedimentos de ensino, além de organizar as atividades de aprendizagem para seus alunos. Dessa forma, a partir do plano de aula, destacamos dois momentos: (1) conteúdo teórico com exemplos e atividades preparados antes da aula e (2) atividades de ensino-aprendizado em sala de aula. Com o aumento da dedicação do professor no momento 1, é esperado que a eficiência no aprendizado do aluno aumente no momento 2. Assim, o momento 2 depende diretamente do momento 1 .

O desenvolvimento proposto neste trabalho é voltado ao preparo das aulas no momento 1. Nesse caso, especificamente, o professor pode utilizar o fluxograma proposto no Tópico (4) 
para compreender o conteúdo e resolver os problemas sobre Análise Combinatória, os quais serão trabalhados em sala de aula. Sugerimos que o professor não utilize o referido fluxograma no ensino-aprendizagem dos alunos (momento 2), mas sim como suporte nas atividades do momento 2 .

Uma metodologia interessante, e que contribui para o desenvolvimento profissional do professor é o Estudo de Aulas (Lesson Study), o qual pode ser resumido em três etapas: planejamento coletivo de uma aula, execução da aula planejada por um professor e observada pelos demais, e reflexão dos pontos fortes sobre a melhora da aula ministrada (MERICHELLI e CURI, 2016). Considerando que a área de ensino problemática é a Combinatória, na primeira etapa do Estudo de Aulas podemos incorporar a proposta desse trabalho, ou seja, no planejamento coletivo considera-se o momento 1 (preparo da aula pelo professor).

O objetivo deste trabalho é propor uma metodologia, utilizando um fluxograma que dê apoio ao professor no momento 1 do planejamento de aula a fim de compreender e resolver problemas de Análise Combinatória. Essa metodologia consiste, resumidamente, em um fluxograma e na construção de conjuntos de agrupamentos cujas propriedades dependem do enunciado do problema de combinatória. A exposição do conteúdo teórico aos alunos, bem como a solução de problemas, deve ser realizada de forma intuitiva e sem utilização de fórmulas. Assim, o fluxograma foi gerado para dar ao professor suporte suficiente no desenvolvimento de sua aula com criatividade e segurança.

No trabalho de Pereira e Bezerra (2015) encontramos um fluxograma correlato ao aqui proposto e, segundo esses autores, a ferramenta pode dinamizar o processo de fixação dos conceitos mais básicos de Análise Combinatória pelos alunos. Contudo, sugerimos que esse procedimento seja utilizado somente pelo professor.

A partir do enunciado de um problema de Análise Combinatória, o fluxograma aqui sugerido fornece o roteiro que indica a solução para cada tipo de problema, respectivamente: combinação simples e com reposição, arranjo simples e com reposição, permutação simples, cíclica e com elementos repetidos. A importância de um procedimento passo a passo para a solução de um problema não é a fórmula em si, mas sim o raciocínio que se deve estabelecer para se resolver tais problemas.

Da mesma maneira que os pais lançam mão de algo como um andador para sua criança com dificuldades em dar seus primeiros passos, sugerimos esse fluxograma a fim de que se possa organizar o raciocínio do aluno frente às informações do enunciado do problema, sem desprezar o aprendizado formal dos conceitos, como precede uma boa didática matemática. 
No Tópico (5), Problemas e resoluções, apresentamos soluções de problemas utilizando a metodologia proposta nesse trabalho. Tais problemas e suas soluções foram retirados dos trabalhos de Santos et al. (2007) e Hazzan e Iezzi (2012). Isso proporcionará ao professor a comparação entre as estratégias de solução apresentadas nesse trabalho com as citadas nas literaturas. Nos três primeiros exemplos de aplicação do método, detalhamos as respectivas resoluções, conforme procedimento mencionado aqui. Nos demais exemplos, aplicamos o método de forma resumida.

\section{COMPREENDENDO O CONJUNTO OBJETO E O ESPAÇO AMOSTRAL}

Neste tópico será exposto os pré-requisitos iniciais para a utilização do fluxograma (Tópico (4)). Antes de utilizar o fluxograma, devemos determinar precisamente o conjunto objeto e o espaço amostral, cujas construções podem ser encontradas na subtópico "Conjunto objeto e espaço amostral". Nos subtópicos "Construindo uma tripla" e "Importância da posição dos elementos na r-upla" mostrar-se-á como gerar o espaço amostral a partir do conjunto objeto. No subtópico "Reposição dos elementos selecionados ao conjunto objeto" abordar-se-á como decidir em quais situações os elementos da $r$-upla retornam ou não ao conjunto objeto.

\subsection{Conjunto objeto e espaço amostral}

Experimentos aleatórios são aqueles que, repetidos em idênticas condições, produzem resultados que não podem ser previstos com clareza, como, por exemplo, quando se lança uma moeda e observar-se a face de cima. O conjunto de todos resultados possíveis de um experimento aleatório, $\Omega$, é chamado de espaço amostral. Usamos as tratativas "experimento aleatório" e "espaço amostral”, pois a Análise Combinatória é aplicada em Probabilidade.

Chamamos de conjunto objeto (CO) ao conjunto que associa letras e/ou números aos objetos usados no experimento aleatório Alguns exemplos de CO: uma moeda com faces $K$ : cara e $C$ : coroa, $A_{1}=\{K, C\}$, um dado, $A_{2}=\{1,2,3,4,5,6\}$, uma fila, $A_{3}=\left\{p_{1}, p_{2}, p_{3}, p_{4}\right\}$, sendo cada $p_{i}$ uma pessoa da fila, um conjunto de camisas, $A_{4}=\left\{c_{1}, c_{2}, c_{3}\right\}$, sendo cada $c_{i}$ uma camisa distinta. 
Sejam os conjuntos objetos $A_{i}=\left\{a_{i 1}, a_{i 2}, \ldots, a_{i m_{i}}\right\}$, para $1 \leq i \leq k, k$ um número qualquer, $k \in \mathbb{N}$, sendo $m_{i}$ o número de elementos (cardinalidade) de cada $A_{i}$, isto também pode ser representado como: \# $A_{i}=m_{i}$. O espaço amostral, $\Omega$, é definido pelo produto cartesiano: $\Omega=A_{1} \times A_{2} \times \ldots \times A_{r}=\left\{\left(x_{1}, x_{2}, \ldots, x_{r}\right) \mid x_{i} \in A_{i}, 1 \leq i \leq r\right\}$, sendo $r \in \mathbb{N}$ um número arbitrário, $r \geq 2$, onde chamamos cada agrupamento $\left(x_{1}, x_{2}, \ldots, x_{r}\right)$ de $r$-upla. Dessa forma, podemos ter uma dupla (2-upla), $\alpha=\left(x_{1}, x_{2}\right)$, uma tripla (3-upla), $\alpha=\left(x_{1}, x_{2}, x_{3}\right)$, uma quádrupla (4-upla), $\alpha=\left(x_{1}, x_{2}, x_{3}, x_{4}\right)$ e, assim, sucessivamente. As posições da $r$-upla são chamadas de coordenadas, então, $x_{1}$ pertence à primeira coordenada ( $1^{\mathrm{a}}$ posição), $x_{2}$ pertence à segunda coordenada ( $2^{\mathrm{a}}$ posição) e, assim por diante. As coordenadas podem estar ordenadas ou não estar ordenadas, conforme será visto no subtópico "Importância da posição dos elementos na r-upla".

Os problemas de Análise Combinatória, estudados neste trabalho, envolvem dois tipos de conjuntos: o(s) conjunto(s) objeto(s), $A_{i}, \quad 1 \leq i \leq k, k \in \mathbb{N}$, e o espaço amostral, $\Omega=A_{1} \times A_{2} \times \ldots \times A_{r}, \quad r \in \mathbb{N}$. No enunciado devem ser dados, explicitamente ou não, a quantidade $k$ e $r$. Por exemplo, "Num baralho de 52 cartas, quatro cartas são escolhidas sucessivamente", neste caso, $k=1$ e $r=4$ são dados explicitamente, pois temos apenas um conjunto objeto, $A_{1}$, constituído de cartas do baralho, e o espaço amostral é $\Omega=A_{1} \times A_{1} \times A_{1} \times A_{1}$. Outro exemplo: “Quantos são os divisores de 72?"; neste caso, $k$ e $r$ são dados implicitamente. Para determiná-los, temos que escrever a decomposição $72=2^{3} 3^{2}$ e o conjunto dos divisores de 72, $D(72)=\left\{2^{n_{1}} 3^{n_{2}} \mid n_{1}=\{0,1,2,3\}, n_{2}=\{0,1,2\}\right\}$. Daí, temos que $k=2$ e $r=2$, pois $A_{1}=\left\{2^{n_{1}} \mid 0 \leq n_{1} \leq 3\right\}=\left\{2^{0}, 2^{1}, 2^{2}, 2^{3}\right\}, A_{2}=\left\{3^{n_{2}} \mid 0 \leq n_{2} \leq 2\right\}=\left\{3^{0}, 3^{1}, 3^{2}\right\}$ e $\Omega=A_{1} \times A_{2}$.

Observamos que $k$ e $r$ são números independentes, os quais participam somente do mesmo problema. Assim, dado um problema de combinatória, logo $k$ está relacionado aos conjuntos objetos $A_{i}, 1 \leq i \leq k$, e $r$ está relacionado com o $r$-upla do espaço amostral $\Omega$. 


\subsection{Construindo uma tripla}

Neste subtópico exemplificar-se-á como construir uma 3-upla, a qual pode ser estendida para uma $r$-upla.

Seja um problema de combinatória, ao qual estão relacionados três conjuntos objetos, ou seja, $k=3$ e $i=1,2,3$, sendo $A_{1}=\left\{a_{11}, a_{12}\right\}, \quad \# A_{1}=m_{1}=2, \quad A_{2}=\left\{a_{21}, a_{22}, a_{23}\right\}$, $\# A_{2}=m_{2}=3$ e $A_{3}=\left\{a_{31}, a_{32}, a_{33}, a_{34}\right\}, \# A_{3}=m_{3}=4$.

O espaço amostral relacionado aos conjuntos objetos acima é dado por: $\Omega=A_{1} \times A_{2} \times A_{3}$, daí construímos uma tripla (agrupamento) qualquer, $x=\left(b_{1}, b_{2}, b_{3}\right) \in \Omega$, como segue:

1. Selecionar, aleatoriamente, um elemento $a_{1 j}, j=1,2$, do conjunto objeto $A_{1}$, e o represente, $b_{1}=a_{1 j}$, na primeira coordenada de $x=\left(b_{1}, \ldots, \ldots\right)$. Este elemento $a_{1 j} \in A_{1}$ pode retornar, ou não, ao conjunto objeto (isso será discutido no subtópico “Reposição dos elementos selecionados ao conjunto objeto");

2. Selecionar, aleatoriamente, um elemento $a_{2 j}, j=1,2,3$, do conjunto objeto $A_{2}$, e o represente, $b_{2}=a_{2 j}$ na segunda coordenada de $x=\left(b_{1}, b_{2}\right.$, $a_{2 j} \in A_{2}$, pode retornar, ou não, ao conjunto objeto;

3. Selecionar, aleatoriamente, um elemento $a_{3 j}, j=1,2,3,4$, do conjunto objeto $A_{3}$, e o represente, $b_{3}=a_{3 j}$ na terceira coordenada de $x=\left(b_{1}, b_{2}, b_{3}\right)$. Este elemento, $a_{3 j} \in A_{3}$, pode retornar, ou não, ao conjunto objeto.

Considerando outro problema de contagem, tal que $k=1$, sendo o conjunto objeto $A=\left\{a_{1}, a_{2}, a_{3}, a_{4}\right\}, \# A=m=4$. Daí, temos outro espaço amostral, $\Omega_{1}=A \times A \times A=A^{3}$, e com isto podemos construir a tripla qualquer, $y=\left(c_{1}, c_{2}, c_{3}\right) \in \Omega_{1}$, como segue:

1. Selecionar, aleatoriamente, um elemento $a_{1}$ do conjunto objeto $A$, e o represente, $c_{1}=a_{1}$, na primeira coordenada de $y=\left(c_{1}, \ldots, \ldots\right)$. Este elemento, $a_{1} \in A$, pode retornar, ou não, ao conjunto; 
2. Selecionar, aleatoriamente, um elemento $a_{2}$ do conjunto objeto $A$, e o represente, $c_{2}=a_{2}$, na segunda coordenada de $y=\left(c_{1}, c_{2}\right.$, _ $)$. Este elemento, $a_{2} \in A$, pode retornar, ou não, ao conjunto objeto;

3. Selecionar, aleatoriamente, um elemento $a_{3}$ do conjunto objeto $A$, e o represente, $c_{3}=a_{3}$ na terceira coordenada de $y=\left(c_{1}, c_{2}, c_{3}\right)$. Este elemento, $a_{3} \in A$, pode retornar, ou não, ao conjunto objeto.

As definições acima podem ser estendidas da tripla para uma $r$-upla qualquer, $\left(b_{1}, b_{2}, \ldots, b_{r}\right)$, sendo $r$ um inteiro não negativo, $r \geq 2$.

\subsection{Importância da posição dos elementos na $r$-upla}

A partir do exemplo do subtópico anterior, construindo uma tripla, sejam $\Omega=A_{1} \times A_{2} \times A_{3}$ e $x=\left(b_{1}, b_{2}, b_{3}\right) \in \Omega$. Dizemos que a posição dos elementos na tripla é importante (ou simplesmente, a posição é importante), quando os elementos selecionados $b_{1}$, $b_{2}$ e $b_{3}$, respectivamente, de $A_{1}, A_{2}$ e $A_{3}$, devem estar ordenados na tripla de acordo com o produto cartesiano $A_{1} \times A_{2} \times A_{3}$. Caso contrário, dizemos que a posição dos elementos na tripla não é importante (ou simplesmente, a posição não é importante), ou seja, quando os elementos selecionados $b_{1}, b_{2}$ e $b_{3}$, respectivamente, de $A_{1}, A_{2}$ e $A_{3}$, não precisam estar ordenados na tripla, de acordo com o produto cartesiano $A_{1} \times A_{2} \times A_{3}$.

Por exemplo, caso possa ocorrer a tripla $x=\left(b_{3}, b_{1}, b_{2}\right), b_{i} \in A_{i}, i=1,2,3$, que não está representada conforme o produto cartesiano $\Omega=A_{1} \times A_{2} \times A_{3}$, temos que a posição não é importante. No caso do produto cartesiano ser da forma $\Omega=A \times A \times A$, são mantidas as noções acima de "posição é importante ou não". As definições acima podem ser estendidas da tripla para uma $r$-upla qualquer, $\left(b_{1}, b_{2}, \ldots, b_{r}\right)$, sendo $r$ um inteiro não negativo, $r \geq 2$. 


\subsection{Reposição dos elementos selecionados ao conjunto objeto}

No exemplo do subtópico "Construindo uma tripla", para $\Omega=A_{1} \times A_{2} \times A_{3}$, considerando o primeiro passo onde selecionamos o elemento $a_{11} \in A_{1}$, temos a representação, $b_{1}=a_{11}$, com isso, a primeira coordenada será $x=\left(a_{11}, \ldots, \ldots\right)$. Daí, após a representação na tripla, o elemento $a_{11}$ pode:

- Retornar ao conjunto objeto, $A_{1}$, de tal forma que ele poderá ser selecionado novamente. Neste caso, este procedimento está relacionado a um problema de combinatória com reposição,

- Não retornar ao conjunto objeto, $A_{1}$, de tal forma que ele não poderá ser selecionado novamente. Neste caso, este procedimento está relacionado a um problema de combinatória sem reposição.

Analogamente ao que fizemos acima, para a primeira coordenada, fazemos para as demais posições da tripla. Assim, os agrupamentos, $\left(b_{1}, b_{2}, b_{3}\right)$, podem estar relacionadas a um problema de combinatória com ou sem reposição. Estas definições podem ser estendidas para toda $r$-upla, $r \geq 2$. No caso de termos um problema sem reposição, diremos que os elementos da $r$-upla são distintos e, para o caso de termos um problema com reposição, diremos que os elementos da $r$-upla são não-distintos (ou indistintos).

\section{PRÉ-REQUISITOS PARA CONSTRUÇÃO DO FLUXOGRAMA}

Neste tópico será exposto o princípio multiplicativo, as características e as fórmulas de cada problema de arranjo, permutação e combinação, completando assim a fundamentação teórica para construção do fluxograma do Tópico (4).

\subsection{Princípio multiplicativo}

Os problemas de combinatórias consistem em determinar a cardinalidade do espaço amostral, isto é, $\# \Omega$.

A seguir será enunciado, em duas partes, A e B, o Princípio Multiplicativo (Princípio Fundamental da Contagem - PFC), cujas determinações das fórmulas e demonstrações de 
teoremas são encontradas no trabalho de Hazzan e Iezzi (2012). O PFC-A considera $k$ conjuntos objetos e está relacionado aos problemas de combinatória com reposição. Já o PFC-B considera apenas um conjunto objeto e está relacionado aos problemas de combinatória sem reposição.

Teorema 1. (PFC - Parte A)

Sejam os conjuntos objetos $A_{i}=\left\{a_{i j_{i}}\right\}$, para $1 \leq i \leq r, \quad r \in \mathbb{N}, \quad r \geq 2, \quad 1 \leq j_{i} \leq m_{i}$, $\# A_{i}=m_{i}$. Se $\Omega_{1}=A_{1} \times A_{2} \times \ldots \times A_{r}=\left\{\left(b_{1}, b_{2}, \ldots, b_{r}\right) \mid b_{i} \in A_{i}, 1 \leq i \leq r\right\}$, sendo as $r$-uplas $\left(b_{1}, b_{2}, \ldots, b_{r}\right)$ relacionadas a um problema de combinatória com reposição, então $\# \Omega_{1}=m_{1} m_{2} \ldots m_{r}$.

A quantidade $\# \Omega_{1}$ apresentada no Teorema 1 é o número total de arranjos com reposição.

Teorema 2. (Princípio Fundamental da Contagem - Parte B)

Seja o conjunto objeto $A=\left\{a_{j}\right\}, \quad 1 \leq j \leq m, \quad \# A=m, \quad r \geq 2 \quad$ e $\quad r \in \mathbb{N}$. Se $\Omega_{2}=\underbrace{A \times A \times \ldots \times A}_{r \text { vezes }}=\left\{\left(b_{1}, b_{2}, \ldots, b_{r}\right) \mid b_{i} \in A, 1 \leq i \leq r\right\}, \quad$ sendo $\quad$ as $\quad r$-uplas $\quad\left(b_{1}, b_{2}, \ldots, b_{r}\right)$ relacionadas a um problema de combinatória sem reposição, então $\# \Omega_{2}=m(m-1)(m-2) \ldots(m-(r-1))=\frac{m !}{(m-r) !}$.

A quantidade $\# \Omega_{2}$, apresentada no Teorema 2, é o número total de arranjos simples (sem reposição).

\subsection{Combinações, arranjos e permutações}

$\operatorname{Dado}(\mathrm{s})$ o(s) conjunto(s) objeto(s) $A_{i}=\left\{a_{i j_{i}}\right\}$, para $1 \leq i \leq s, s \in \mathbb{N}, s \geq 1,1 \leq j_{i} \leq m_{i}$, sendo \# $A_{i}=m_{i}$. Os problemas de combinatória são chamados de combinação, arranjo e permutação. Tais problemas estão relacionados aos respectivos espaços amostrais, $\Omega$, e a $r$ upla, $\left(b_{1}, b_{2}, \ldots, b_{r}\right) \in \Omega$, sendo $r \geq 2$. Conforme o tipo de problema de combinatória são formados agrupamentos, representados pelas $r$-uplas. Desta forma, desejamos saber a quantidade de $r$-uplas, isto é, a cardinalidade do espaço amostral, $\# \Omega$. Para tal, seguem abaixo 
as definições, fórmulas e as características desses problemas, conforme os trabalhos de Hazzan e Iezzi (2012) e Santos, Mello e Murari (2007).

1) Arranjo com reposição.

Os problemas de combinatória com as seguintes características:

- com reposição

- a posição de $b_{i}$ na $r$-upla é importante e

- os elementos do conjunto objeto são distintos

são denominados arranjos com reposição. Assim, o número de $r$-uplas, formadas pelos $m_{1}, m_{2}, \ldots, m_{r}$ elementos de $A_{1}, A_{2}, \ldots, A_{r}$, é dado por (Teorema 1 ):

$$
\# \Omega=A R^{m_{1} m_{2} \ldots m_{r}}=m_{1} m_{2} \ldots m_{r} .
$$

2) Arranjo simples.

Os problemas de combinatória com as seguintes características:

- sem reposição,

- a posição de $b_{i}$ na $r$-upla é importante e

- os elementos do conjunto objeto são distintos

são denominados arranjos simples. Assim, o número de $r$-uplas, formadas pelos $m$ elementos de $A$, é dado por (Teorema 2):

$$
\# \Omega=A_{r}^{m}=\frac{m !}{(m-r) !} .
$$

3) Combinação simples.

Os problemas de combinatória com as seguintes características:

- sem reposição,

- posição dos elementos $b_{i}$ na $r$-upla não é importante e

- os elementos do conjunto objeto são distintos

são denominados combinação simples.

Sejam o conjunto objeto $A=\left\{a_{j}\right\}, 1 \leq j \leq m$, \#A=m e o espaço amostral: $\Omega=A^{r}=$ $\left\{\left(b_{1}, b_{2}, \ldots, b_{r}\right) \mid b_{i} \in A, \quad 1 \leq i \leq r\right\}$,

sendo $r \geq 2$ e $r \in \mathbb{N}$. Assim, o número de $r$-uplas, formadas pelos $m$ elementos de $A$, é dado por: 


$$
\# \Omega=C_{r}^{m}=\frac{m !}{(m-r) ! r !} .
$$

4) Combinação com repetição.

Os problemas de combinatória com as seguintes características:

- com reposição,

- posição dos elementos $b_{i}$ na $r$-upla não é importante e

- existem elementos repetidos no conjunto objeto

são denominados combinação com repetição.

Sejam o conjunto objeto $A=\left\{a_{j}\right\}, 1 \leq j \leq m, \# A=m$ e o espaço amostral:

$$
\Omega=A^{r}=\left\{\left(b_{1}, b_{2}, \ldots, b_{r}\right) \mid b_{i} \in A, \quad 1 \leq i \leq r\right\},
$$

sendo $r \geq 2$ e $r \in \mathbb{N}$. Assim, o número das $r$-uplas, formadas pelos $m$ elementos de $A$, é dado por:

$$
\# \Omega=C R_{r}^{m}=C_{m}^{r+m-1}=\frac{(r+m-1) !}{(r-1) ! m !} .
$$

5) Permutação simples.

Os problemas de combinatória com as seguintes características:

- sem reposição,

- a posição de $b_{i}$ na $r$-upla é importante,

- os elementos do conjunto objeto são distintos e

- o número de elementos da $r$-upla é igual ao número de elementos do conjunto objeto são denominados permutação simples.

Sejam o conjunto objeto $A=\left\{a_{j}\right\}, 1 \leq j \leq m, \# A=m$ e o espaço amostral:

$$
\Omega=A^{m}=\left\{\left(b_{1}, b_{2}, \ldots, b_{m}\right) \mid b_{i} \in A, \quad 1 \leq i \leq m\right\} .
$$

Assim, o número de $r$-uplas, formadas pelos $m$ elementos de $A$, é dado por:

$\# \Omega=P_{m}=m !$.

6) Permutação circular.

Os problemas de combinatória com as seguintes características:

- sem reposição,

- a posição de $b_{i}$ na $r$-upla é importante,

- os elementos do conjunto objeto são distintos, 
- o número de elementos da $r$-upla é igual ao número de elementos do conjunto objeto,

- os elementos do conjunto objeto podem ser representados em uma circunferência são denominados permutação circular.

Sejam o conjunto objeto $A=\left\{a_{j}\right\}, 1 \leq j \leq m, \# A=m$ e o espaço amostral:

$$
\Omega=A^{m}=\left\{\left(b_{1}, b_{2}, \ldots, b_{m}\right) \mid b_{i} \in A, \quad 1 \leq i \leq m\right\} .
$$

Assim, o número de $r$-uplas, formadas pelos $m$ elementos de $A$, é dado por:

$$
\# \Omega=P C_{m}=(m-1) ! .
$$

7) Permutação com elementos repetidos:

Os problemas de combinatória com as seguintes características:

- sem reposição,

- a posição de $b_{i}$ na $r$-upla é importante,

- o número de elementos da $r$-upla é igual ao número de elementos do conjunto objeto e

- existem elementos repetidos no conjunto objeto

são denominados Permutação com Elementos Repetidos.

Sejam o conjunto objeto:

$$
A=\{\underbrace{a_{11}, a_{12}, \ldots, a_{1 n_{1}}}_{n_{1} \text { elementos repatidos }}, \underbrace{a_{21}, a_{22}, \ldots, a_{2 n_{2}}}_{n_{2} \text { elementos repatidos }}, \ldots, \underbrace{a_{k 1}, a_{k 2}, \ldots, a_{k n_{k}}}_{n_{k} \text { elementos repatidos }}, \underbrace{b_{1}, b_{2}, \ldots, b_{n}}_{n \text { elementos distintos }}\},
$$

$\# A=m=n_{1}+n_{2}+\ldots+n_{k}+n$, daí $m-n_{k}=n_{1}+n_{2}+\ldots+n$, e o espaço amostral:

$$
\Omega=A^{m}=\left\{\left(x_{1}, x_{2}, \ldots, x_{m}\right) \mid x_{i} \in A, 1 \leq i \leq m\right\} .
$$

Assim, a permutação com $n_{1}, n_{2}, \ldots, n_{k}$ elementos repetidos é dada por:

$$
\# \Omega=C_{m-n_{k}}^{m} P_{m-n_{k}}^{n_{1} n_{2} \ldots n_{k-1}}=P_{m}^{n_{1} n_{2} \ldots n_{k}}=\frac{m !}{n_{1} ! n_{2} ! \ldots n_{k} !} .
$$

\section{FLUXOGRAMA}

Conforme o objetivo deste trabalho, o fluxograma é a ferramenta que dará suporte ao professor, quando estiver resolvendo problemas de Análise Combinatória. A sua construção se baseia nos conteúdos contidos no Tópico (3), Pré-Requisitos para Construção do Fluxograma, que estabelece, além das fórmulas, as características de cada tipo de problema. 
Tabela 1. Símbolos e os respectivos significados utilizados no fluxograma.

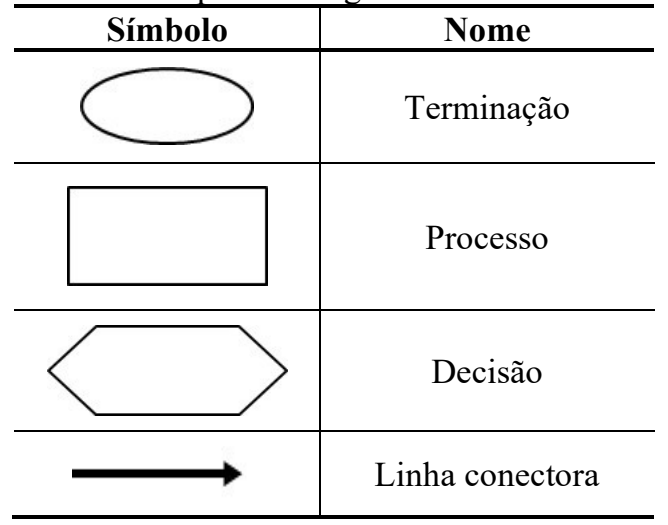

Fonte: Elaborada pelo autor (2020)

$\mathrm{Na}$ Tabela 1 temos os símbolos utilizados no fluxograma da Figura 1. Assim, o significado de cada símbolo é (ASCENCIO; CAMPOS, 2012):

- Terminação: indica o início ou o fim de um fluxo no diagrama de processos,

- Processo: indica um determinado processo e suas funções e atividades,

- Decisão: indica que uma decisão (Falso (Não) ou Verdadeiro (Sim)) terá que ser tomada e que o fluxo do processo seguirá determinada direção em função dessa decisão e

- Linha conectora: é um símbolo de conexão que serve para indicar uma interligação entre dois outros símbolos e a direção do fluxo.

No fluxograma (Figura 1) todas as linhas conectoras estão numeradas de 1 até 21 e, a partir da linha 3, esta numeração é acompanhada da decisão $S=S i m$ ou N=Não.

Para resolver cada problema de combinatória, temos uma e, somente uma, sequência de números, conforme indicada na Tabela 2. A linha conectora numerada por 1 , sempre se liga ao início do fluxograma e as linhas 6, 10,12,14, 16, 19 e 21 sempre se ligam ao fim do fluxograma. Cada uma das linhas da Tabela 2 é chamada de solução única. Existem problemas de combinatória, cujas soluções necessitam da utilização de duas ou mais linhas da Tabela 2 . Nestes casos, as resoluções dos problemas são chamadas de soluções mistas. Assim, uma solução mista é constituída por duas ou mais soluções únicas. No Tópico (5), Problemas e resoluções, serão apresentados exemplos de problemas para estes tipos de soluções. 


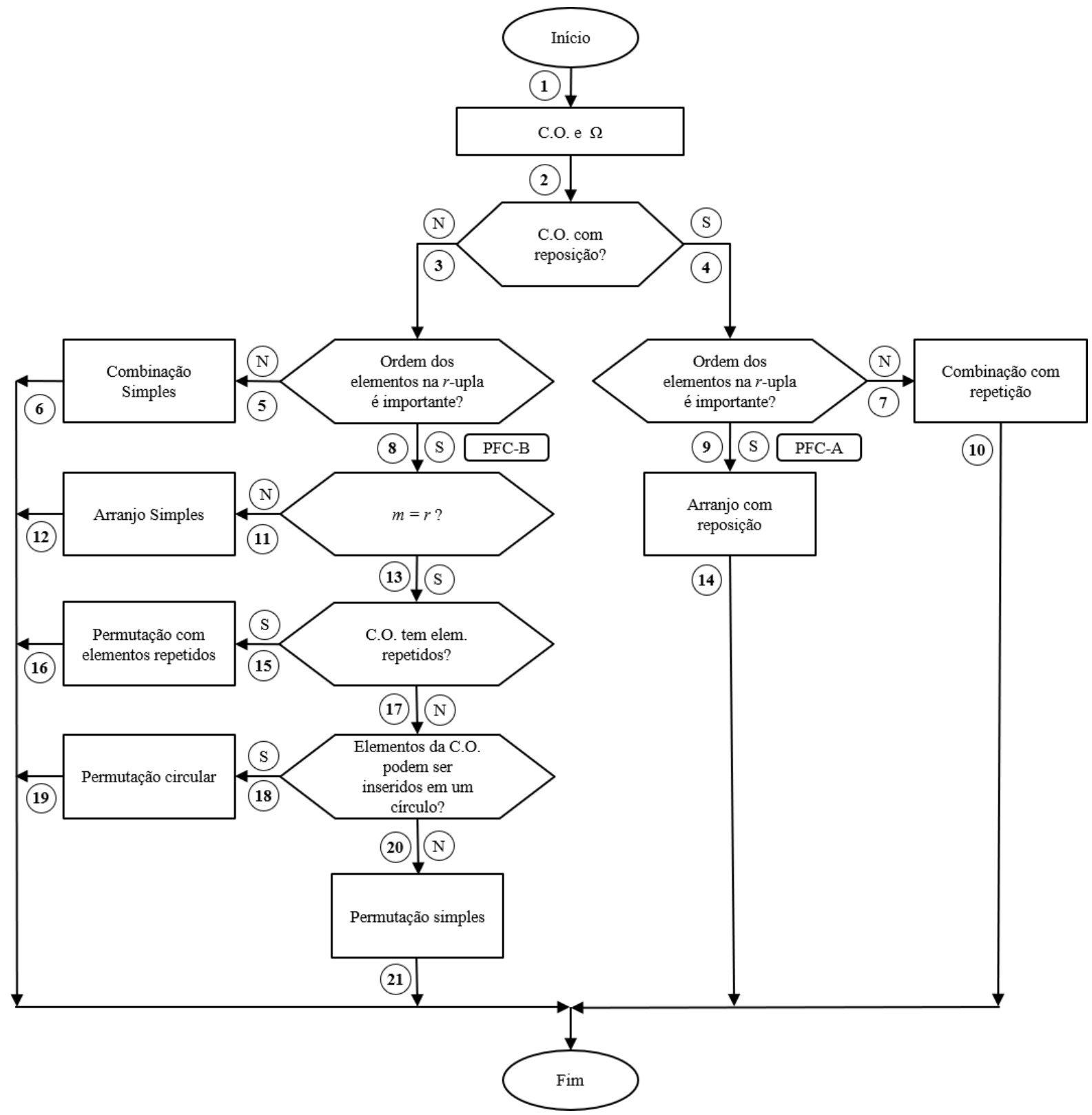

Figura 1 - Fluxograma proposto para resolver problemas de combinação, sendo dado inicialmente o conjunto objeto, C.O., e o espaço amostral, $\Omega$.

Fonte: Elaborada pelo autor (2020)

O processo entre as linhas 1 e 2 tem fundamental importância na solução dos problemas, pois, após a compreensão do enunciado do problema, sempre devemos determinar (ou descrever) o conjunto objeto, $\mathrm{CO}$, e o espaço amostral, $\Omega$, conforme Tópico (2), Compreendendo o conjunto objeto e o espaço amostral.

Tabela 2. A partir da Figura 1, obtemos as sequências de números das linhas conectoras (ou soluções únicas) e as fórmulas para resolver problemas de combinatória.

\begin{tabular}{l|c|c}
\hline \multicolumn{1}{c|}{ Problema de análise combinatória } & Números das linhas conectoras & Fórmula \\
\hline Arranjo com reposição & $1,2,4,9$ e 14. & Equação (1) \\
\hline Arranjo simples & $1,2,3,8,11$ e 12. & Equação (2) \\
\hline Combinação simples & $1,2,5$ e 6. & Equação (3) \\
\hline
\end{tabular}




\begin{tabular}{l|c|l}
\hline Combinação com repetição & $1,2,4,7$ e 10. & Equação (4) \\
\hline Permutação simples & $1,2,3,8,13,17,20$ e 21. & Equação (5) \\
\hline Permutação circular & $1,2,3,8,13,17,18$ e 19. & Equação (6) \\
\hline Permutação com elementos repetidos & $1,2,3,8,13,15$ e 16. & Equação (7) \\
\hline
\end{tabular}

Fonte: Elaborada pelo autor (2020)

\section{PROBLEMAS E RESOLUÇÕES}

Neste tópico serão apresentados alguns problemas de Análise Combinatória, bem como as respectivas soluções. O fluxograma da Figura 1 será utilizado nestas soluções. Resolver um problema de análise combinatória é determinar, $\# \Omega$.

Os problemas de combinatória de baixa e média dificuldade serão resolvidos, já que pretendemos mostrar ao professor o processo sugerido no fluxograma. Nesse contexto, baixa dificuldade está relacionada à solução única do problema e média dificuldade à solução mista do problema. Os problemas sobre: arranjo simples, arranjo com reposição e combinação simples foram resolvidos detalhadamente e os demais problemas foram resolvidos de forma resumida. Esses problemas foram retirados dos trabalhos de Santos et al. (2007) e Hazzan e Iezzi (2012).

\subsection{Arranjo simples}

Problema: Quantos números telefônicos, com 7 dígitos distintos, podem ser formados se usarmos os dígitos de 0 a 9 ?

\section{Solução única:}

(i) Seja o conjunto objeto (CO): $A=\{0,1,2,3,4,5,6,7,8,9\}$, conjunto dos dígitos de 0 até 9, sendo: $\# A=m=10$.

(ii) Para construir o espaço amostral, $\Omega$, a partir do $\operatorname{CO} A$, observamos no enunciado que os números de telefones devem ter 7 dígitos, assim:

$$
\Omega=A \times A \times A \times A \times A \times A \times A=A^{7}=\{\underbrace{\left(a_{1}, a_{2}, \ldots, a_{7}\right)}_{7 \text {-upla }} \mid a_{i} \in A, 1 \leq i \leq 7\} .
$$

O evento sugerido no enunciado é tomar números do $\mathrm{CO} A$ e gerar 7-uplas de tal forma que os números dentro de cada 7-upla ocorram apenas uma vez (7 dígitos distintos), caracterizando que os elementos selecionados não retornam para o $\mathrm{CO}$ (sem reposição). 
(iii) Considerando os Itens (i) e (ii), utilizando o fluxograma (Figura 1) e a Tabela 2, a solução do problema é dada pela seguinte sequência de números das linhas conectoras: 1, 2, 3, 8, 11 e 12, que representa a solução de um problema de Arranjo Simples. Com isso, temos a fórmula dada pela Equação (2) e, portanto, a solução do problema é:

$\# \Omega=A_{r}^{m}=A_{7}^{10}=\frac{10 !}{(10-7) !}=604.800$,

ou seja, existem 604.800 números de telefones com 7 dígitos distintos.

\subsection{Arranjos simples e arranjo com reposição}

Problema: Existem duas urnas. A primeira urna contém quatro bolas numeradas de 1 a 4 e a segunda urna contém três bolas numeradas de 7 a 9 . Duas bolas são extraídas da primeira urna, sucessivamente e sem reposição, e, em seguida, duas bolas são extraídas da segunda urna, sucessivamente e sem reposição. Quantos números de quatro algarismos são possíveis formar nestas condições?

Solução mista:

i) Sejam os conjuntos objetos (CO): $U_{1}=\{1,2,3,4\}$, conjunto de bolas da primeira urna, $\# U_{1}=m_{1}=4$, e $U_{2}=\{7,8,9\}$, conjunto de bolas da segunda urna, $\# U_{2}=m_{2}=3$.

ii) A partir do enunciado, temos que extrair duas bolas de cada urna. Assim, se considerarmos o espaço amostrar, do tipo $\Omega=U_{1} \times U_{1} \times U_{2} \times U_{2}$, não será possível resolver o problema, conforme as condições dadas no enunciado. Daí temos que separar o problema em duas partes, onde devemos calcular os seguintes espaços amostrais:

$\Omega_{1}=U_{1} \times U_{1}=\{\underbrace{\left(a_{1}, a_{2}\right)}_{\text {2-upla }} \mid a_{1}, a_{2} \in U_{1}\}$, conjunto das 2-uplas de bolas retiradas da

primeira urna, sendo $r_{1}=2 \mathrm{e}$,

$$
\Omega_{2}=U_{2} \times U_{2}=\{\underbrace{\left(b_{1}, b_{2}\right)}_{\text {2-upla }} \mid b_{1}, b_{2} \in U_{2}\} \text {, conjunto das 2-uplas de bolas retiradas da }
$$

segunda urna, sendo $r_{2}=2$. 
O espaço amostrar $\Omega_{1}$ é formado, após retirar da primeira urna, de duas bolas sucessivamente e estas não retornam ao conjunto objeto. Por se tratar de uma fila que vai compor um número de dois algarismos, a ordem dos elementos na 2-upla, $\left(a_{1}, a_{2}\right)$, é importante. Analogamente, o espaço amostrar $\Omega_{2}$ é formado, após retirar da segunda urna, duas bolas sucessivamente e estas não retornam ao conjunto objeto. Por se tratar de uma fila que vai compor um número de dois algarismos, a ordem dos elementos na 2-upla, $\left(b_{1}, b_{2}\right)$, é importante.

iii) Considerando os Itens (i) e (ii), utilizando o fluxograma (Figura 1) e a Tabela 2, temos as seguintes sequências de números das linhas conectoras:

- Primeira urna: 1, 2, 3, 8, 11 e 12, que representa um problema de Arranjo Simples, cuja fórmula é dada pela Equação (2). Portanto a solução é: $\# \Omega_{1}=A_{r_{1}}^{m_{1}}=A_{2}^{4}=\frac{4 !}{(4-2) !}=12$,

ou seja, existem 12 números de dois algarismos formados pelos elementos da primeira urna.

- Segunda urna: 1, 2, 3, 8, 11 e 12, que representa um problema de Arranjo Simples, cuja fórmula é dada pela Equação (2). Portanto a solução é:

$\# \Omega_{2}=A_{r_{2}}^{m_{2}}=A_{2}^{3}=\frac{3 !}{(3-2) !}=6$,

ou seja, existem 6 números de dois algarismos formados pelos elementos da segunda urna.

iv) No enunciado solicitou-se determinar quantos números de quatro algarismos são possíveis formar conforme as condições dadas no Item (iii). Logo, temos que gerar números de 4 algarismos, ou seja, 4-uplas compostas da seguinte forma: $\left(\gamma_{1}, \gamma_{2}\right)$, sendo $\gamma_{1} \in \Omega_{1}$ e $\gamma_{2} \in \Omega_{2}$. Desta forma, consideramos $\Omega_{1}$ e $\Omega_{2}$ sendo conjuntos objetos (CO). Daí, construímos o seguinte espaço amostral:

$\Omega=\Omega_{1} \times \Omega_{2}=\{\underbrace{\left(\gamma_{1}, \gamma_{2}\right)}_{\text {2-upla }} \mid \gamma_{1} \in \Omega_{1}$ e $\gamma_{2} \in \Omega_{2}\}=\{\underbrace{\left(\gamma_{1}, \gamma_{2}\right)}_{\text {2-upla }} \mid \gamma_{1} \in \Omega_{1}$ e $\gamma_{2} \in \Omega_{2}\}$,

ou seja, o conjunto das 2-uplas de bolas retiradas da primeira e segunda urna, sendo $r=2$. Embora os elementos de $\Omega$ sejam compostos por 2-uplas, cada elemento desta 2-upla é composto por dois algarismos, formando os 4 algarismos solicitado no 
problema. Além disso, cada elemento da 2 -upla, $\left(\gamma_{1}, \gamma_{2}\right)$, podem retornar aos conjuntos objetos $\Omega_{1}$ e $\Omega_{2}$.

v) Considerando o Itens (iv), utilizando o fluxograma (Figura 1) e a Tabela 2, temos a seguinte sequência de números das linhas conectoras: 1, 2, 4, 9 e 14, que representa a solução de um problema de Arranjo com reposição, cuja fórmula é dada pela Equação (1). Portanto, a solução é:

$$
\# \Omega=A R^{\# \Omega_{1}, \# \Omega_{2}}=\# \Omega_{1} \# \Omega_{2}=(12)(6)=72,
$$

ou seja, existem 72 números de 4 algarismos formados pelos elementos da primeira e segunda urnas.

\subsection{Combinação simples e arranjo com reposição}

Problema: Temos 10 homens e 10 mulheres. Quantas comissões de 5 pessoas podem ser formadas, se em cada uma delas houver 3 homens e 2 mulheres?

Solução mista:

i) $\mathrm{CO}$ :

- $H=\left\{a_{1}, a_{2}, \ldots, a_{10}\right\}$, conjunto de homens, sendo $\# H=m_{1}=10$, e

- $M=\left\{b_{1}, b_{2}, \ldots, b_{10}\right\}$, conjunto de mulheres, sendo $\# M=m_{2}=3$.

ii) Espaços Amostrais:

- $\Omega_{1}=H \times H \times H=\{\underbrace{\left(x_{1}, x_{2}, x_{3}\right)}_{3 \text {-upla }} \mid x_{1}, x_{2}, x_{3} \in H\}:$ conjunto das 3-uplas de homens retirados de $H$, sendo $r_{1}=3$,

- $\Omega_{2}=M \times M=\{\underbrace{\left(y_{1}, y_{2}\right)}_{\text {-upla }} \mid y_{1}, y_{2} \in M\}:$ conjunto das 2-uplas de mulheres retiradas de $M$, sendo $r_{2}=2$ e

- $\Omega=\Omega_{1} \times \Omega_{2}=\{\underbrace{\left(\alpha_{1}, \alpha_{2}\right)}_{\text {2-upla }} \mid \alpha_{1} \in \Omega_{1}$ e $\alpha_{2} \in \Omega_{2}\}:$ conjunto das 2-uplas de homens e mulheres retirados, respectivamente de $\Omega_{1}$ e $\Omega_{2}$, sendo $r=2$.

iii) Cálculo de $\# \Omega_{1}$ e $\# \Omega_{2}$ 
- Com relação aos espaços amostrais $\Omega_{1}$ e $\Omega_{2}$, os elementos não retornam aos respectivos COs,

- Não importa a ordem das escolhas dos elementos de $\Omega_{1}$ e $\Omega_{2}$,

- Utilizando o fluxograma Figura 1 e a Tabela 2, temos as seguintes sequências de números das linhas conectoras:

○ Para $\Omega_{1}: 1,2,5$ e 6, que representa a solução de um problema de Combinação Simples, cuja fórmula é dada pela Equação (3), portanto, a solução é:

$\# \Omega_{1}=C_{r_{1}}^{m_{1}}=C_{3}^{10}=\frac{10 !}{(10-3) ! 3 !}=120$,

ou seja, existem 120 comissões de três homens.

○ Para $\Omega_{2}: 1,2,5$ e 6, que representa a solução de um problema de Combinação Simples, cuja fórmula é dada pela Equação (3), portanto, a solução é:

$\# \Omega_{2}=C_{r_{21}}^{m_{2}}=C_{2}^{10}=\frac{10 !}{(10-2) ! 2 !}=45$,

ou seja, existem 45 comissões de duas mulheres.

iv) Cálculo de $\# \Omega$

- Os elementos que retornam ao cada elemento da 2-upla, $\left(\alpha_{1}, \alpha_{2}\right)$, podem retornar, respectivamente, aos conjuntos objetos $\Omega_{1}$ e $\Omega_{2}$.

- Utilizando o fluxograma Figura 1 e a Tabela 2, temos as seguintes sequências de números das linhas conectoras: 1, 2, 4, 9 e 14, que representa a solução de Arranjo com reposição, cuja fórmula é dada pela Equação (1), portanto, a solução é:

$$
\# \Omega=A R^{\# \Omega_{1}, \# \Omega_{2}}=\left(\# \Omega_{1}\right)\left(\# \Omega_{2}\right)=(120)(45)=5400,
$$

ou seja, existem 5400 comissões formadas por 3 homens e duas mulheres.

\subsection{Combinação com repetição}

Problema: De quantos modos diferentes podemos distribuir 10 bombons idênticos em 4 caixas diferentes?

Solução única:

$\mathrm{CO}: A=\{\underbrace{b, b, \ldots, b}_{10 \text { vezes }}\}, \# A=m=10$. 
Espaço amostral: $\Omega=A^{r}=\left\{\left(b_{1}, b_{2}, b_{3}, b_{4}\right) \mid b_{i} \in A, \quad 1 \leq i \leq 4\right\}, r=4$.

Pelo enunciado temos as seguintes características:

- com reposição,

- posição dos elementos $b_{i}$ na 4-upla não é importante,

- existem elementos repetidos no conjunto objeto $A$.

Pela Figura 1 e a Tabela 2, temos a seguinte sequência de números das linhas conectoras: 1, 2, 4, 7 e 10. Assim, a quantidade de maneiras diferentes de distribuir os bombons nas caixas é dada por (Equação (4)):

$$
\# \Omega=C R_{3}^{4}=C_{10}^{13}=\frac{(4+10-1) !}{(4-1) ! 10 !}=\frac{13 !}{3 ! 10 !}=286 .
$$

\subsection{Permutação simples}

Problema: Nomeando-se 6 cidades por $A, B, C, D, E$ e $F$ determine o número de maneiras que permitem a ida de $A$ para $F$, passando por todas demais cidades.

Solução única:

$\mathrm{CO}: A=\{B, C, D, E\}, \# A=m=4$.

Espaço amostral: $\Omega=A^{4}=\left\{\left(A, x_{1}, x_{2}, x_{3}, x_{4}, F\right) \mid x_{i} \in A, 1 \leq i \leq 4\right\}, r=4$.

Pelo enunciado, temos as seguintes características:

- sem reposição,

- a posição de $x_{i}$ na $r$-upla é importante,

- o número de elementos da $r$-upla é igual ao número de elementos do conjunto objeto.

Pela Figura 1 e a Tabela 2, temos a seguinte sequência de números das linhas conectoras:

$1,2,3,8,13,17,20$ e 21 . Assim, o número de maneiras que se pode ir da cidade A para cidade F é dada por (Equação (5)):

$$
\# \Omega=P_{4}=4 !=24 \text {. }
$$

\subsection{Permutação circular}

Problema: De quantas formas 4 pessoas podem se sentar ao redor de uma mesa circular? Solução única: 
$\mathrm{CO}: A=\left\{p_{1}, p_{2}, p_{3}, p_{4}\right\}, \# A=m=4$.

Espaço amostral: $\Omega=A^{4}=\left\{\left(x_{1}, x_{2}, x_{3}, x_{4}\right) \mid x_{i} \in A, \quad 1 \leq i \leq 4\right\}, r=4$.

Pelo enunciado, temos as seguintes características:

- sem reposição,

- a posição de $x_{i}$ na 4-upla é importante,

- o número de elementos da 4-upla é igual ao número de elementos do conjunto objeto e

- os elementos do CO podem ser inseridos em um círculo.

Pela Figura 1 e a Tabela 2, temos a seguinte sequência de números das linhas conectoras: $1,2,3,8,13,17,18$ e 19. Assim, a quantidade de formas com que 4 pessoas podem se sentar ao redor de uma mesa circular é dada por (Equação (6)):

$$
\# \Omega=P C_{4}=(4-1) !=3 !=6 \text {. }
$$

\subsection{Permutação com repetição}

Problema: Se um time de futebol jogou 13 partidas em um campeonato, tendo perdido 5 jogos, empatado 2 e vencido 6 jogos, de quantos modos pode isto ter ocorrido?

Solução única:

$\mathrm{CO}: A=\{\underbrace{d_{1}, d_{2}, \ldots, d_{5}}_{n_{1}=5 \text { :derrotas }}, e_{n_{2}=2: \text { empates }}, \underbrace{v_{1}, v_{2}, \ldots, v_{6}}_{n_{3}=6: \text { vitórias }}\}, \quad \# A=m=13$, com $n_{1}=5, n_{2}=2$ e $n_{3}=6$.

Espaço amostral: $\Omega=A^{13}=\left\{\left(x_{1}, x_{2}, \ldots, x_{13}\right) \mid x_{i} \in A, \quad 1 \leq i \leq 13\right\}, r=13$.

Pelo enunciado temos as seguintes características:

- sem reposição,

- a posição de $x_{i}$ na 13 -upla é importante,

- o número de elementos da13-upla é igual ao número de elementos do conjunto objeto e

- existem elementos repetidos no conjunto objeto.

Pela Figura 1 e a Tabela 2, temos a seguinte sequência de números das linhas conectoras: $1,2,3,8,13,15$ e 16 . Assim, a quantidade de modos com que um time de futebol, o qual 
disputou 13 partidas, dentre as quais perdeu 5 jogos, empatou 2 jogos e venceu 6 jogos é (Equação (7)):

$$
\# \Omega=P_{13}^{5,2,6}=\frac{13 !}{5 ! 2 ! 6 !}=36.036
$$

\section{CONCLUSÕES}

Muitos autores relatam as dificuldades dos professores com relação ao tema Análise Combinatória e defendem que muitos chegam a evitar ou até a não abordar esse conteúdo. Quase sempre o ensino se limita ao treinamento no uso de fórmulas e algoritmos para encontrar o número de arranjos, combinações ou permutações, sem proporcionar que os alunos derivem as referidas fórmulas pelo uso da manipulação dos elementos dos espaços amostrais. A dificuldade do professor aumenta ao se resolver os problemas sem o uso das fórmulas.

Ao estruturar um plano de aula para Análise Combinatória, o professor pode se sentir seguro e minimizar a sua ansiedade. Este plano é apenas um roteiro, um instrumento de referência, é esquemático e deve ser personificado pelo professor, enriquecendo-o e conferindo a ele sua dinâmica própria.

A partir do plano de aula, observamos dois momentos: (1) conteúdo teóricos, exemplos e atividades preparados antes da aula e (2) atividades de ensino-aprendizado em sala de aula. Com isso, esperamos que o aumento da dedicação do professor no momento 1 aumentará a eficiência no momento 2 .

Foram apresentados nas Seções (2) e (3) os pré-requisitos que deram suporte à construção do fluxograma de apoio ao professor, o qual é um procedimento facilitador para o professor durante o momento 1. Esse fluxograma, aliado a outros elementos, tais como, o conjunto objeto e o espaço amostral, proporcionam a compreensão da teoria envolvendo combinatória, bem como a resolução de problemas. Observamos que o professor geralmente não utiliza este procedimento no momento 2 (em sala de aula), considerando-o apenas como suporte no momento 1.

Finalmente, como exemplo ilustrativo ao professor, apresentamos e resolvemos, por meio do referido fluxograma, diversos problemas, a saber: Arranjo com reposição, Arranjo simples, Combinação simples, Combinação com repetição, Permutação simples, Permutação circular, Permutação com elementos repetidos. 
REFERÊNCIAS

EVES, Howard. Introdução à história da matemática. 5. ed. Campinas: Ed. Unicamp, 2011.

BOYER, Carl B.; MERZBACH, Uta C. História da matemática. 3. ed. São Paulo, Ed. Edgard Blücher, 2012.

SILVA, Alex Sandro Vaz. Atividades escolares que envolvem a análise combinatória, a partir da expectativa do desenvolvimento da habilidade de contagem, segundo a BNCC. 2020. 90 f. Dissertação (Mestrado) - Universidade Federal de São Paulo, campus de São Jose dos Campos, Instituto de Ciência e Tecnologia. Programa de Pós-Graduação em Matemática em Rede Nacional (PROFMAT). Disponível em: https://sca.profmatsbm.org.br/sca v2/get tcc3.php?id=170870231. Acesso em: 06 jan. 2021.

BRASIL. Lei n. ${ }^{\circ}$ 9394/96. Lei de Diretrizes e Bases da Educação Nacional. Estabelece as diretrizes e bases da educação nacional. Brasília: 1996. Disponível em: https://www2.senado.leg.br/bdsf/bitstream/handle/id/529732/lei_de_diretrizes_e_bases_1ed.p df. Acesso em: 27 nov. 2020.

BRASIL. Ministério da Educação. Base Nacional Comum Curricular. Brasília: 2018. Disponível em: http://basenacionalcomum.mec.gov.br/images/BNCC_EI_EF_110518_versaofinal_site.pdf. Acesso em: 27 nov. 2020.

MOREIRA, Francis Miller Barbosa; MAGINA, Sandra Maria Pinto. A análise combinatória na formação de professores: um Estudo Diagnóstico. VII CIBEM - Congresso Iberoamericano de Educação Matemática. Actas del VII CIBEM, 2013. Disponível em: http://funes.uniandes.edu.co/17606/1/Barbosa2013A.pdf. Acesso em: 27 nov. 2020.

SABO, Ricardo Deszo. Análise de livros didáticos do ensino médio: um estudo dos conteúdos referentes à combinatória. 2007, 54 f. Dissertação (Especialização) - Centro Universitário Fundação Santo André, Santo André. Disponível em: https://www.pucsp.br/ cileda/Monografia RicardoSabo.pdf. Acesso em: 27 nov. 2020.

SCHMIDT, Wilian, MARIANI, Rita de Cássia Pistóia. Raciocínio combinatório: uma metaanálise com ênfase nas representações semióticas. Revista de Ensino de Ciências e Matemática (REnCiMa). v. 8, n. 3, p. 109-128, 2017. Disponível em: http://revistapos.cruzeirodosul.edu.br/index.php/rencima/article/view/1196/899. Acesso em: 27 nov. 2020.

CARVALHO, Paulo Cezar Pinto. Métodos de contagem e probabilidade. Rio de Janeiro: IMPA, 2015. Disponível em: http://www.obmep.org.br/docs/apostila2.pdf. Acesso em: 27 nov. 2020.

MERICHELLI, Marco Aurélio Jarreta; CURI, Edda. Estudos de aula (“Lesson Study”) como metodologia de formação de professores. Revista de Ensino de Ciências e Matemática (REnCiMa). V. 7, n. 4, p. 15-27, 2016. Disponível em: 
http://revistapos.cruzeirodosul.edu.br/index.php/rencima/article/view/1202/838. Acesso em: 27 nov. 2020.

PEREIRA, André Gustavo Campos; BEZERRA, José Rauryson Alves. Uma ferramenta para ajudar na fixação dos conceitos básicos de análise combinatória. Revista do Centro de Ciências Naturais e Exatas - UFSM, v. 37, Ed. Especial PROFMAT, p. 503-510, 2015. Disponível em: https://periodicos.ufsm.br/cienciaenatura/article/download/14659/pdf. Acesso em: 27 nov. 2020.

SANTOS, José Plínio O.; MELLO, Margarida P.; MURARI, Idani T. C. Introdução à analise combinatória. Rio de Janeiro: Ed. Ciência Moderna, 2007.

\section{NOTAS}

\section{AGRADECIMENTOS}

Não se aplica.

FINANCIAMENTO

Não se aplica.

\section{CONTRIBUIÇÕES DE AUTORIA}

Resumo/Abstract/Resumen: Antonio Luís Venezuela

Introdução: Antonio Luís Venezuela

Referencial teórico: Antonio Luís Venezuela

Análise de dados: Antonio Luís Venezuela

Discussão dos resultados: Antonio Luís Venezuela

Conclusão e considerações finais: Antonio Luís Venezuela

Referências: Antonio Luís Venezuela

Revisão do manuscrito: Antonio Luís Venezuela

Aprovação da versão final publicada: Antonio Luís Venezuela

\section{CONFLITOS DE INTERESSE}

O autor declara não haver nenhum conflito de interesse de ordem pessoal, comercial, acadêmico, político e financeiro referente a este manuscrito.

\section{DISPONILIDADE DE DADOS DE PESQUISA}

Informo que os dados foram apresentados no próprio artigo.

\section{CONSENTIMENTO DE USO DE IMAGEM}

Não se aplica.

\section{APROVAÇÃO DO COMITÊ DE ÉTICA EM PESQUISA}

Não se aplica.

\section{COMO CITAR - ABNT}

VENEZUELA, Antonio Luís. Análise combinatória: metodologia de apoio ao professor. REAMEC - Rede Amazônica de Educação em Ciências e Matemática. Cuiabá, v. 9, n. 1, e21015, janeiro-abril, 2021. DOI: 10.26571/reamec.v9i1.10440.

\section{COMO CITAR - APA}

Venezuela, A. L. (2021). Análise combinatória: metodologia de apoio ao professor. REAMEC-Rede Amazônica de Educação em Ciências e Matemática, 9 (1), e21015. DOI: 10.26571/reamec.v9i1.10440. 


\section{LICENCA DE USO}

Licenciado sob a Licença Creative Commons Attribution-NonCommercial 4.0 International (CC BY-NC 4.0). Esta licença permite compartilhar, copiar, redistribuir o manuscrito em qualquer meio ou formato. Além disso, permite adaptar, remixar, transformar e construir sobre o material, desde que seja atribuído o devido crédito de autoria e publicação inicial neste periódico.

\section{DIREITOS AUTORAIS}

Os direitos autorais são mantidos pelos autores, os quais concedem à Revista REAMEC - Rede Amazônica de Educação em Ciências e Matemática - os direitos exclusivos de primeira publicação. Os autores não serão remunerados pela publicação de trabalhos neste periódico. Os autores têm autorização para assumir contratos adicionais separadamente, para distribuição não exclusiva da versão do trabalho publicada neste periódico (ex.: publicar em repositório institucional, em site pessoal, publicar uma tradução, ou como capítulo de livro), com reconhecimento de autoria e publicação inicial neste periódico. Os editores da Revista têm o direito de proceder a ajustes textuais e de adequação às normas da publicação.

\section{PUBLISHER}

Universidade Federal de Mato Grosso. Programa de Pós-graduação em Educação em Ciências e Matemática (PPGECEM) da Rede Amazônica de Educação em Ciências e Matemática (REAMEC). Publicação no Portal de Periódicos UFMT. As ideias expressadas neste artigo são de responsabilidade de seus autores, não representando, necessariamente, a opinião dos editores ou da referida universidade.

\section{EDITOR}

Dailson Evangelista Costa

\section{HISTÓRICO}

Submetido: 18 de maio de 2020.

Aprovado: 17 de setembro de 2020.

Publicado: 27 de janeiro de 2021. 